\title{
A detailed aerosol mixing state model for investigating interactions between mixing state, semivolatile partitioning, and coagulation
}

\author{
J. Lu ${ }^{1, *}$ and F. M. Bowman ${ }^{2}$ \\ ${ }^{1}$ Department of Chemical Engineering, Vanderbilt University, Nashville, Tennessee, USA \\ ${ }^{2}$ Department of Chemical Engineering, University of North Dakota, Grand Forks, North Dakota, USA \\ * now at: Planning and Technical Support Division, California Air Resources Board, Sacramento, California, USA
}

Received: 3 December 2009 - Published in Atmos. Chem. Phys. Discuss.: 11 January 2010

Revised: 18 April 2010 - Accepted: 19 April 2010 - Published: 29 April 2010

\begin{abstract}
A new method for describing externally mixed particles, the Detailed Aerosol Mixing State (DAMS) representation, is presented in this study. This novel method classifies aerosols by both composition and size, using a userspecified mixing criterion to define boundaries between compositional populations. Interactions between aerosol mixing state, semivolatile partitioning, and coagulation are investigated with a Lagrangian box model that incorporates the DAMS approach. Model results predict that mixing state affects the amount and types of semivolatile organics that partition to available aerosol phases, causing external mixtures to produce a more size-varying composition than internal mixtures. Both coagulation and condensation contribute to the mixing of emitted particles, producing a collection of multiple compositionally distinct aerosol populations that exists somewhere between the extremes of a strictly external or internal mixture. The selection of mixing criteria has a significant impact on the size and type of individual populations that compose the modeled aerosol mixture. Computational demands for external mixture modeling are significant and can be controlled by limiting the number of aerosol populations used in the model.
\end{abstract}

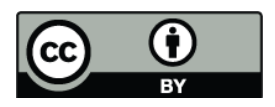

Correspondence to: F. M. Bowman (frank.bowman@und.edu)

\section{Introduction}

Aerosol impacts on health, visibility, climate, and cloud formation, are governed by particle composition, size, and mixing state. For example, particles of different composition have been shown to exhibit different cloud condensation nuclei (CCN) behavior (Broekhuizen et al., 2004; Corrigan and Novakov, 1999) and Lesins et al. (2002) found that optical properties influencing radiative forcing for internal and external mixtures of black carbon and ammonium sulfate could differ by over $50 \%$. In order to correctly assess the effects of atmospheric aerosols it is essential to know their composition and mixing state and understand how these properties change with time.

Many measurement studies have shown that particles emitted from different sources have distinct compositions (Rogge et al., 1993; Chow et al., 2004). Thus, aerosol emissions enter the atmosphere as an external mixture of compositionally distinct populations. However, aerosol mixing state is not constant, but evolves over time. As particles interact with each other and with the gas phase, they become more compositionally similar and resemble an internal mixture. Single particle measurements have helped illuminate this transition from external to internal mixing. Hasegawa and Ohta (2002), for example, found that in urban areas, where emissions sources are nearby, soot particles were externally mixed, but in non-urban areas, where particles had undergone long range transport, internal mixtures of soot and water soluble materials were more common. Naoe and Okada (2001) examined aerosol mixing state at an urban site and found that the number fraction of internally mixed particles increased with particle size (2-12\% internally mixed for

Published by Copernicus Publications on behalf of the European Geosciences Union. 
$0.02-0.03 \mu \mathrm{m}$ particles vs. $17-74 \%$ for the $0.1-0.2 \mu \mathrm{m}$ size range), indicative of mixing due to particle growth.

These changes in aerosol mixing state are most likely due to secondary aerosol formation. Semivolatile products formed in the gas phase are able to partition to any particles present in the atmosphere. Over time this has the effect of adding secondary aerosol mass to initially distinct populations, making them more similar in composition. Additionally, semivolatile compounds will continue to repartition, maintaining a balance between different aerosol populations via equilibrium with a common gas phase, even as new particles are emitted.

Aerosol mixing state is also influenced by coagulation. When particles of different compositions coalesce the resulting particle will be a mixture of the two original compositions. The actual degree of mixing that occurs depends on the relative sizes of the coagulating particles. If one particle is much smaller than the other, the coagulated particle will have a composition very similar to that of the original large particle. Significant mixing only occurs when the colliding particles are of similar size. Jacobson calculated that for moderately polluted conditions, most emitted particles will become internally mixed to some degree within a few hours due to coagulation (Jacobson, 2002).

Just as partitioning can influence mixing state, mixing state can have a large effect on how semivolatile partitioning occurs. Kleeman et al. (1997) have shown that with semivolatile inorganics a bimodal size distribution can be created due to hygroscopic particles growing more quickly than hydrophobic particles of similar initial size as relative humidity increases. Such bimodal distributions are observed in atmospheric measurements, but could not be reproduced by assuming an internal mixture. They also found differences in the amount of aerosol nitrate formed for internal and external mixtures. Similar behavior occurs with semivolatile organics, where polar organics will partition preferentially to an aqueous aerosol phase, while nonpolar organics will be absorbed mainly by a more nonpolar organic aerosol phase (Griffin et al., 2003; Pun et al., 2002).

Traditionally, air quality models have treated aerosols as an internal mixture. Unfortunately, assuming an incorrect mixing state can artificially distort the aerosol size and component distribution (FassiFihri et al., 1997; Kleeman et al., 1997; Jacobson, 2001, 2002). Recently, other approaches for describing external mixtures have been used in models developed by Kleeman (Kleeman et al., 1997; Bhave et al., 2002; Ying et al., 2004; Ying et al., 2007), Jacobson (Jacobson, 2001, 2002), and Riemer et al. (2009). The Kleeman source-oriented external mixture trajectory model represents the aerosol with thousands of particle classes according to particle size, emission source category, and time of emission. These distinct populations interact with the same gas phase, but evolve independently based on their unique compositions. The model does not account for coagulation, which was considered to have a negligible effect on aerosol mass distributions for urban time scales. When considering aerosol number or ultrafine particles, however, coagulation is a significant process that must be included (Binkowski and Roselle, 2003; Jacobson, 1997a; Jung and Kim, 2006; Yao et al., 2005).

Jacobson's approach describes the aerosol with several single source populations that interact with the gas phase and with each other to form additional mixed composition populations. Over time, emissions are added to the source populations, and when coagulation or condensation occurs, mass is transferred to the appropriate mixed population. In accounting for coagulation it places all binary mixtures into the same distribution, regardless of the relative amounts of each component. As a result, the mixed population containing components $\mathrm{A}$ and $\mathrm{B}$ will include both particles that have 99\% A, 1\% B and particles that have 1\% A, 99\% B.

The PartMC model developed by Riemer et al. (2009) is a particle-resolved Monte Carlo model that tracks the composition of individual particles within a single cell Lagrangian framework. It accounts for condensation and coagulation involving a population of $\sim 10^{5}$ particles, with coagulation treated using a new efficient stochastic method. With such a high level of detail it is computationally demanding, but provides a numerical benchmark against which lower resolution mixing state models can be compared.

This paper presents a new modeling method for representing externally mixed particles. The detailed aerosol mixing state (DAMS) representation classifies particles by both size and composition, providing a level of compositional detail that can distinguish between external and internal mixtures. The DAMS approach, along with corresponding coagulation and condensation routines, is incorporated into a box model and used in simulations of a simplified atmospheric scenario. Model predictions of aerosol size and component distributions are compared between internal and external mixtures to demonstrate the impact of mixing state on aerosol growth and evolution. Simulations with and without coagulation, and using different mixing state criteria are also compared to further illustrate the interactions between aerosol mixing state, semivolatile partitioning, and coagulation.

\section{Model formulation}

\subsection{Model framework}

The model and simulations in this study were designed to provide a simple scenario for testing the new aerosol mixing state method and for initial investigations of the interactions between aerosol mixing state, semivolatile organic partitioning, and coagulation. A Lagrangian box model was used to simulate the evolution of aerosol size and composition distributions over time as semivolatile organics partition onto emitted organic and inorganic particles, particles coagulate with each other, and particles and gases are removed 
by dilution with clean air. The model is an extension of previous aerosol models (Bowman et al., 1997; Bowman and Melton, 2004; Sheehan and Bowman, 2001; Bowman and Karamalegos, 2002), with updated treatments of the aerosol representation method and the coagulation process. Future studies based on results of this work are planned with more complex models capable of representing atmospheric scenarios in greater detail.

A key feature of the model is a new representation of the aerosol distribution which classifies particles into discrete bins based on both size and composition, with sufficient resolution to distinguish between different compositional mixtures. Condensational growth and coagulation routines have also been developed for use with the new aerosol representation. Adapted from Jacobson (1997b; 2002), these approaches move particles into the appropriate size and compositional bins as their size and/or composition change due to condensation of semivolatile vapors and coagulation with other particles. The mathematical details of these novel methods are described in the following sections.

\subsection{Detailed aerosol mixing state representation}

To better represent externally mixed particles, a new sectional approach, the detailed aerosol mixing state (DAMS) representation, has been developed that categorizes compositional mixtures into different populations such that particles are divided into discrete bins based on both size and composition. This new scheme is similar to that used by Jacobson $(2001,2002)$, but expanded to provide greater compositional detail. A unique population exists for every possible combination of aerosol mixture components. For example, in a ternary system of components A, B, and C, there would be 7 populations as shown in Fig. 1. Within the DAMS representation, a component can be either an individual chemical compound or multiple compounds that are grouped together.

An important feature of the DAMS representation is that it can distinguish between particles that are truly mixed, i.e., containing significant fractions of different components, and ones that, while technically a mixture, contain only small amounts of other components. For each aerosol component a threshold mass fraction, $c_{Q}$, is specified that defines what is considered a significant amount of an individual component. For the 3-component system in Fig. 1, if the threshold is set at $5 \%$ for all components, particles in the $\mathrm{AB}$ population will contain at least $5 \% \mathrm{~A}$ and $5 \% \mathrm{~B}$, but less than $5 \% \mathrm{C}$. Particles in the $\mathrm{A}, \mathrm{B}$, or $\mathrm{C}$ populations will be mostly a single component, but may also contain small amounts (below the specified threshold) of other components.

The DAMS approach can be applied to aerosol mixtures with any number of components. In general, for a mixture with $N_{C}$ components the total number of aerosol populations, $N_{P}$, will be

$N_{P}=2^{N_{C}}-1$

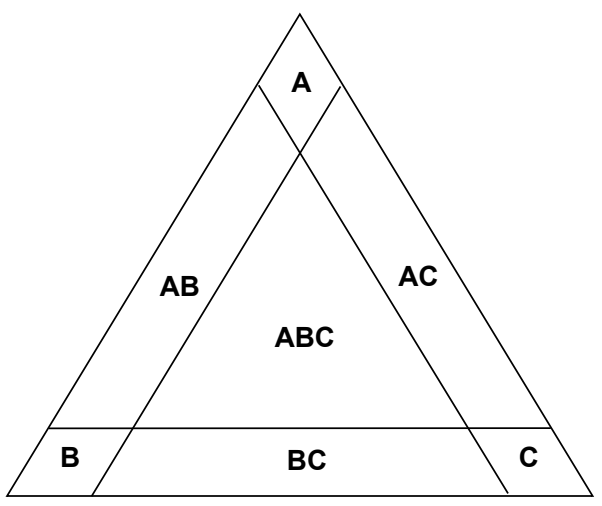

Fig. 1. Compositional categories for a ternary aerosol system.

The populations can be conveniently ordered and defined by specifying the index number of a given population, $N$, as

$N=\sum_{Q=1}^{N_{C}} 2^{Q-1} g(Q)$

where $g(Q)$, a function indicating if the mass fraction of component $Q, x_{Q}$, exceeds the specified mixing threshold, $c_{Q}$, is defined as

$g(Q)=\left\{\begin{array}{l}1 \text { when } x_{Q} \geq c_{Q} \\ 0 \text { when } x_{Q}<c_{Q}\end{array}\right.$

To ensure that a population exists for all possible compositions, the individual mixing threshold values are subject to the constraint that $\Sigma c_{Q} \leq 1$. Following the notation of Jacobson (1994), the total volume concentration of particles in bin $N k$, corresponding to population type $N$ and particle size range $k$ is denoted by $v_{N k}$, and the volume concentration of component $Q$ in that bin is denoted by $v_{Q, N k}$. The total, component, and compound mass concentrations are denoted by $m_{N k}, m_{Q, N k}$ and $m_{q, N k}$. Similarly, the particle diameter and number concentration in that bin are described as, respectively, $D_{p N k}$ and $n_{N k}$. Thus, with the DAMS representation, an externally mixed aerosol is divided into bins that are classified by both composition and size. Within the computer model, aerosol particles are defined by the variables $D_{p N k}$ and $m_{q, N k}$. Because particles are assumed spherical with constant density, specification of diameter and mass also defines the number and volume concentration. When needed for model subroutines, $n_{N k}$ and $v_{q, N k}$ are calculated from $D_{p N k}$ and $m_{q, N k}$.

\subsection{Condensation}

Condensational growth occurs in the model due to gasparticle partitioning of semivolatile vapors. Semivolatile organic partitioning is modeled as an absorption process 
(Pankow, 1994a, b), and is described by a partitioning coefficient, $K_{q}$,

$K_{q}=\frac{m_{q}}{M_{t} G_{q}}$

which is the ratio of the aerosol-phase mass concentration, $m_{q}$, and the gas-phase mass concentration, $G_{q}$, of semivolatile compound $q$, normalized by the total mass concentration of absorbing material, $M_{t}$. As shown by Pankow (1994b), the absorptive partitioning coefficient can be expressed as a function of physical and thermodynamic properties of the semivolatile compound

$$
K_{q}=\frac{R T}{\mathrm{MW} \gamma_{q} p_{q}^{o}}
$$

where $R$ is the ideal gas constant, $T$ is temperature, MW is the mean molecular weight of the absorbing aerosol phase, $\gamma_{q}$ is the activity coefficient of compound $q$ in this phase, and $p_{q}^{\circ}$ is the vapor pressure of compound $q$ as a pure liquid.

The temperature and composition dependence of the partitioning coefficient for compound $q$ in bin $N k, K_{q, N k}$, is described in the model using an expression that determines $K_{q, N k}$ at a given set of $T, \mathrm{MW}_{N k}$, and $\gamma_{q, N k}$ conditions relative to a reference value of the partitioning coefficient, $K_{q}{ }^{*}$, at a set of reference $T^{*}, \mathrm{MW}^{*}$, and $\gamma_{q}{ }^{*}$ conditions (Bowman and Karamalegos, 2002)

$$
\begin{aligned}
& K_{q, N k}= \\
& K_{q}^{*}\left(\frac{\mathrm{MW}^{*}}{\mathrm{MW}_{N k}}\right)\left(\frac{\gamma_{q}^{*}}{\gamma_{q, N k}}\right)\left(\frac{T}{T^{*}}\right) \exp \left[\frac{H_{q}}{R}\left(\frac{1}{T}-\frac{1}{T^{*}}\right)\right]
\end{aligned}
$$

where $H_{q}$ is the enthalpy of vaporization of compound $q$.

Mass transfer between gas and particle phases is calculated using a dynamic partitioning approach. The mass flux $J_{q, N k}$ of semivolatile compound $q$ to particles in bin $N k$ is written as (Wexler and Seinfeld, 1990)

$J_{q, N k}=n_{N k} \frac{4 \pi R_{p N k} D_{q}}{1+\frac{\lambda}{\alpha_{q} R_{p N k}}}\left(G_{q}-G_{q, N k}^{e q}\right)$

where $n_{N k}$ is the number concentration of particles in bin $N k, R_{p N k}$ is the particle radius in bin $N k, D_{q}$ is the gasphase diffusivity of compound $q, \lambda$ is the air mean free path, and $\alpha_{q}$ is the accommodation coefficient of species $q$ on the particle. $G_{q}$ is the concentration of $q$ in the bulk gas phase, and $G_{q, N k}^{e q}$ is the gas-phase concentration of compound $q$ in equilibrium with aerosol particles in bin $N k$, which is calculated from Eq. (4)

$G_{q, N k}^{e q}=\frac{m_{q, N k}}{M_{t, N k} K_{q, N k}}$

where $m_{q, N k}$ is the aerosol phase concentration of compound $q$ in bin $N k, M_{t, N k}$ is the total absorbing aerosol mass concentration in bin $N k$, and $K_{q, N k}$ is the partitioning coefficient defined by Eq. (6) for compound $q$ in bin $N k$.
The model treats condensation and evaporation by first calculating gas-particle partitioning and then, if necessary, moving particles to the appropriate size and composition bin. Changes in particle and gas concentrations are determined by solving the coupled set of differential equations

$$
\begin{aligned}
& \frac{d m_{q, N k}}{d t}=J_{q, N k} \\
& \frac{d G_{q}}{d t}=\sum_{N k} J_{q, N k}
\end{aligned}
$$

with $J_{q, N k}$ calculated from Eqs. (6)-(8). The coupled set of differential equations in Eqs. (9) and (10) is solved in the current version of the model using the LSODES sparse matrix ODE solver (Hindmarsh, 1983). For the simulations in this study, absolute and relative error tolerance values were set at $10^{-8}$ and $10^{-2}$, respectively.

When condensational growth causes the aerosol to move in composition and size space, aerosol mass and number need to be transferred to the appropriate bin. For this study we have used the moving center approach (Jacobson, 1997a) where the characteristic volume of a bin $u_{N k}$, is calculated from the total aerosol volume and number within the bin

$u_{N k}=\frac{v_{N k}}{n_{N k}}$

and is allowed to vary between the fixed size boundaries of the bin. Similarly the aerosol composition within a bin is allowed to vary between the fixed compositional boundaries of the bin defined by the mixing thresholds $c_{Q}$. When particle composition and/or size change beyond the defined limits of a given bin, all particles in the bin are transferred to the correct bin for their new size and/or composition. The total particle number, $n_{N k}$, and compound volumes, $v_{q, N k}$, from the original bin are added to those already present in the new bin and a new characteristic size and composition for the bin are calculated. With this approach both total particle number and total particle volume are conserved. And since both sets of particles have a size and composition that lie within the boundaries of the bin, when they are combined together the resulting average size and composition will also be within the bin boundaries.

\subsection{Coagulation}

Coagulation will also cause particles to move in composition and size space. Unlike the coagulation of an internal mixture, where only particle size is a concern, the coagulation process for an external mixture is much more complex. Coagulation combines particles with different sizes and compositions, and the resulting aerosol must be assigned to both the correct size section and the correct compositional population.

The coagulation scheme used here is based on the approach developed by Jacobson (1997b, 2002). Coagulation is permitted to occur between all of the size and composition bins. The coagulated particle size will not, in general, 
be exactly equal to the particle size of any bin, but will fall somewhere between the particle sizes of two adjacent size bins. When this occurs, a fraction of the coagulated aerosol is assigned to each of these two destination size bins. This is done in such a way that the particle sizes in both the source and destination bins do not change. In the source bins, aerosol volume and number are reduced, and in the destination bins they are increased, with total aerosol volume being conserved, and total aerosol number reduced consistent with the loss of particle number due to coagulation.

A key difference between our coagulation procedure and that used by Jacobson is the manner in which coagulated aerosol is assigned to a compositional population. In the work of Jacobson, particles are only allowed to move to more internally mixed populations. For example, when particles from population $\mathrm{A}$ and from population $\mathrm{B}$ coagulate, the resulting particle will be assigned to the mixed population $\mathrm{AB}$, which gains mass of both $\mathrm{A}$ and $\mathrm{B}$. Aerosol from a mixed population will never be moved to a less mixed population. With the DAMS approach, however, it is possible for aerosol to move in both directions between more and less well mixed populations. For example, when a very small particle from population $\mathrm{AB}$ coagulates with a much larger particle from population $\mathrm{A}$, the newly coagulated particle may only contain a small amount of $\mathrm{B}$. If the concentration of $\mathrm{B}$ is below the mixing threshold, then the newly formed particle will be assigned to population $\mathrm{A}$, adding mass of both $\mathrm{A}$ and $\mathrm{B}$ to the population. As will be discussed below, this has important implications in how the coagulation equations are solved.

The mathematical formulation for calculating coagulation in DAMS follows from previously developed coagulation models (Jacobson et al., 1994; Seinfeld and Pandis, 1998; Jacobson, 1997a, 2002), with adaptations to account for the expanded representation of aerosol mixing state. The volume concentration of compound $q$ in a bin for population type $N$ and particle size range $k$ at time $t, v_{q, N k, t}$, can be written using the notation of Jacobson as:

$$
\begin{aligned}
& v_{q, N k, t}= \\
& \frac{v_{q, N k, t-\Delta t}+\frac{1}{2} \Delta t \sum_{L=1}^{N_{P}} \sum_{M=1}^{N_{P}} \sum_{j=1}^{k} \sum_{i=1}^{k-1} f_{L i, M j, N k, t} \beta_{L i, M j, t} v_{q, L i, t} n_{M j, t}}{1+\Delta t \sum_{L=1}^{N_{P}} \sum_{i=1}^{N_{S}}\left(1-f_{L i, N k, N k, t}\right) \beta_{L i, N k, t} n_{L i, t}}
\end{aligned}
$$

where $v_{q, N k, t-\Delta t}$ is the volume concentration of compound $q$ in bin $N k$ for population $N$ and size $k$ at time $t-\Delta t, \Delta t$ is the time step size, $f_{L i, M j, N k, t}$ is the fraction of the particle volume formed by coagulation between bins $L i$ and $M j$ that is assigned to bin $N k$ at time $t, \beta_{L i, M j, t}$ is the coagulation kernel between bins $L i$ and $M j$ at time $t$, and $N_{S}$ is the total number of particle sizes.

The volume fraction $f_{L i, M j, N k, t}$ is defined as (Jacobson, 1997b)

$$
\begin{aligned}
& f_{L i, M j, N k, t}= \\
& \left\{\begin{array}{ccc}
\left(\frac{u_{N k+1, t}-V_{L i, M j, t}}{u_{N k+1, t}-u_{N k, t}}\right) \frac{u_{N k, t}}{V_{L i, M j, t}} & u_{N k, t} \leq V_{L i, M j, t}<u_{N k+1, t} & k<N_{S} \\
1-f_{L i, M j, N k-1, t} & u_{N k-1, t}<V_{L i, M j, t}<u_{N k, t} & k>1 \\
1 & V_{L i, M j, t} \geq u_{N k, t} & k=N_{S} \\
0 & \text { all other cases } &
\end{array}\right.
\end{aligned}
$$

where $V_{L i, M j, t}=u_{L i, t}+u_{M j, t}$ is the volume of a single particle created from coagulation of a particle from bin $L i$ and a particle from bin $M j$. The volume fraction in Eq. (13) divides the volume of a coagulated particle between two adjacent size bins, with each portion taking on the characteristic volume of the destination bin. As a result, the characteristic volumes for each bin do not change during the coagulation calculation. Equation (13) also ensures that total aerosol volume is conserved and that the reduction in total aerosol number is consistent with the rate of coagulation.

Brownian diffusion is assumed the dominant coagulation mechanism in this study, with the coagulation kernel $\left(\beta \mathrm{m}^{3}\right.$ particle $\left.{ }^{-1} \mathrm{~s}^{-1}\right)$ calculated based on Fuchs equation (Jacobson, 1997a; Seinfeld and Pandis, 1998). The total volume concentration in bin $N k$ at time $t, v_{N k, t}$, is the sum of the individual compound values

$v_{N k, t}=\sum_{q=1}^{N_{C}} v_{q, N k, t}$

and the number concentration of particles in bin $N k, n_{N k, t}$, is calculated from the total volume concentration and the characteristic single particle volume in the bin

$n_{N k, t}=\frac{v_{N k, t}}{u_{N k, t}}$

Because particles do not always move to more mixed populations, a noniterative semi-implicit approach where coagulation to and from less mixed populations is calculated before more-mixed populations (Jacobson, 2002), can not be used. Instead, Eq. (12) is fully implicit and is solved iteratively. The volume, $v_{N k, t}$, and number, $n_{N k, t}$, concentrations for each bin are updated by Eqs. (14) and (15) using volume and number concentrations from the previous iteration. Values from the previous time step are used as initial guesses for the first iteration. For this study iterations were continued until the relative difference in volume concentration between two consecutive iterations was less than $1 \%$ for all bins and the change in total volume concentration for the entire system was less than $0.001 \%$. These convergence criteria values were selected to provide a balance of accuracy and calculation speed for a relatively simple aerosol system. Work is ongoing to improve computational efficiency of the entire coagulation code and to determine optimum convergence criteria for more complex and computationally demanding aerosol systems. 


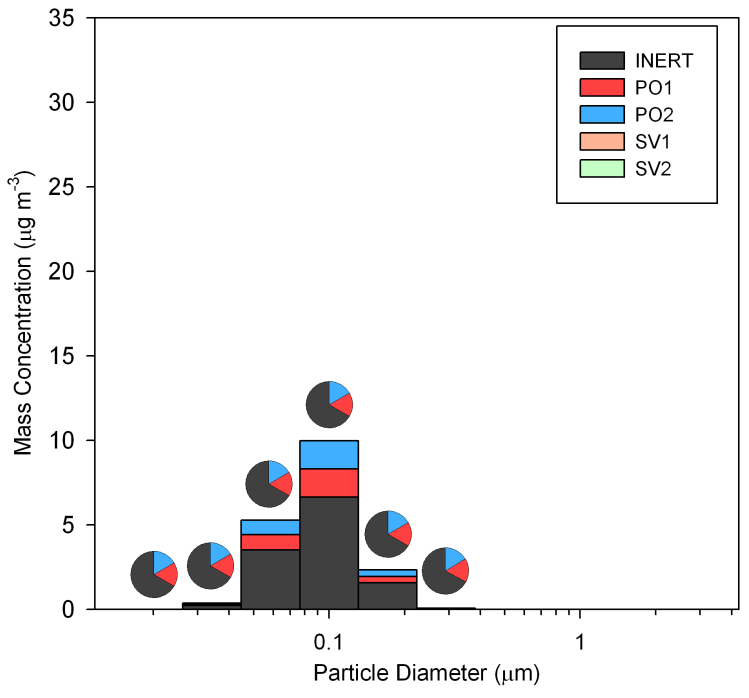

Fig. 2. Emitted and initial aerosol distribution for model simulations.

\section{Model simulations}

Model simulations were performed using a simplified base case scenario, patterned after a polluted urban environment. While sufficient detail was included to test the new aerosol mixing state method and to enable a qualitative investigation of mixing state, partitioning, and coagulation interactions, it is important to note that this scenario is not meant to represent specific atmospheric conditions.

Simulation parameters for the base case scenario are summarized in Table 1. Primary particulate (PO1, PO2, INERT) and semivolatile vapor (SV1, SV2) compounds are emitted at a constant rate into the model volume. Particles are emitted with the size distribution shown in Fig. 2. Dilution of the air parcel with clean air removes particles and semivolatile vapors at a constant rate of $5.0 \% \mathrm{hr}^{-1}$. Gas-particle mass transfer calculations assume an accommodation coefficient of unity. Temperature varies with a fixed diurnal cycle ranging from 15 to $35^{\circ} \mathrm{C}$. Emission rates were specified so as to produce a total fine particle concentration of approximately $60 \mu \mathrm{g} \mathrm{m}^{-3}$, with approximately $30 \mu \mathrm{g} \mathrm{m}^{-3}$ of organic aerosol. Together, the constant emissions, constant dilution, and diurnal temperature cycle result in a repeating steady state after the model has run for several days. To capture the full evolution of all particle distributions, the system was simulated for a $188 \mathrm{~h}$ period, and model results from the end of the simulation were analyzed. Additional simulation time did not noticeably change the daily cycles of bulk aerosol mass, number, and composition or its distribution among the different size and composition bins.

A five component mixture was used to represent the aerosol phase in the base case scenario. In this scenario, each component represents a single model compound, such that components correspond directly to the five model com-
Table 1. Model parameters for base case scenario.

\begin{tabular}{ll}
\hline Particulate emission rates $\left(\mu \mathrm{g} \mathrm{m}^{-3} \mathrm{~h}^{-1}\right)$ & PO1: 0.375 \\
& PO2: 0.375 \\
& INERT: 1.5 \\
Semivolatile emission rates $\left(\mu \mathrm{g} \mathrm{m}^{-3} \mathrm{~h}^{-1}\right)$ & SV1: 1.5 \\
& SV2: 1.0 \\
& 5.0 \\
Air parcel dilution rate $\left(\% \mathrm{~h}^{-1}\right)$ & 1.0 \\
Accommodation coefficient & $25 \pm 10$ \\
Temperature $\left({ }^{\circ} \mathrm{C}\right)$ & 188 \\
Simulation time $(\mathrm{h})$ & 0.1 \\
Mixing criterion &
\end{tabular}

pounds. The compounds and their partitioning properties are listed in Table 2. Primary organic aerosol is represented by heneicosane and levoglucosan, which are key compounds from diesel soot (Schauer et al., 1999; Chow et al., 2007) and wood smoke (Schauer et al., 2001) emissions, respectively. Heneicosane, model compound PO1, is nonpolar whereas levoglucosan, model compound $\mathrm{PO} 2$, is relatively polar. $\mathrm{PO} 1$ and $\mathrm{PO} 2$ are both assumed to be nonvolatile and remain almost entirely in the aerosol phase. Semivolatile organic aerosol is represented in the scenario by heptadecanoic acid, SV1, and norpinonic acid, SV2, compounds with relatively low and high polarity, respectively. One general inert compound, that is nonvolatile and does not interact with the organic compounds, is used to represent all other aerosol constituents. The highly simplified representation of aerosol composition chosen for this initial study provides a relatively small number of mixture populations and allows the general qualitative behavior of the system to be more clearly seen. Future studies targeted at simulating realistic atmospheric conditions will more accurately describe atmospheric aerosol composition by including individual inorganic compounds, water, and other organics, and will better account for the interactions between these compounds.

The aerosol distribution can be simulated as either an internal or an external mixture. In all cases, the size distribution uses 10 size sections, with mean diameters logarithmically spaced from 0.02 to $2.5 \mu \mathrm{m}$. For an internal mixture, there is a single well-mixed aerosol population throughout the entire simulation. The initial population, shown in Fig. 2, has a peak near $0.09 \mu \mathrm{m}$ and contains PO1, PO2, and INERT, with total concentration of $18.0 \mu \mathrm{g} \mathrm{m}^{-3}$. The initial mixture is one third organic, with equal amounts of PO1 and PO2. Particulate emissions during the simulation have the same composition and size distribution as the initial distribution.

For an external mixture, multiple aerosol populations are simulated, each with different size and compositional distributions. With 5 components in the base case, there are a total of 31 possible compositional populations. The initial distribution contains only three populations, pure PO1, pure PO2 and pure INERT. Summed together, these populations 
Table 2. Aerosol compound properties.

\begin{tabular}{llllll}
\hline Model Name & Compound & $K_{i}^{*}\left(\mathrm{~m}^{3} \mu \mathrm{g}^{-1}\right)$ & $H_{i}\left(\mathrm{~kJ} \mathrm{~mol}^{-1}\right)$ & $\mathrm{MW}_{i}\left(\mathrm{~g} \mathrm{~mol}^{-1}\right)$ & Relative Polarity \\
\hline PO1 & Heneicosane & $1.0 \times 10^{12}$ & 0.0 & 296 & Low \\
PO2 & Levoglucosan & $1.0 \times 10^{12}$ & 0.0 & 162 & High \\
SV1 & Heptadecanoic acid & 0.0172 & 75 & 270 & Low \\
SV2 & Norpinonic acid & 0.0420 & 75 & 170 & High \\
INERT & & & & \\
\hline
\end{tabular}

$K_{i}^{*}$ values correspond to reference conditions of $T^{*}=298 \mathrm{~K}, \mathrm{MW}^{*}=150 \mathrm{~g} \mathrm{~mol}^{-1}, \gamma_{i}^{*}=1$.

Table 3. Total number, mass, and compound concentrations at $t=188 \mathrm{~h}$ for base case scenario with external and internal mixtures, with and without coagulation.

\begin{tabular}{lllll}
\hline & \multicolumn{2}{c}{ With Coagulation } & \multicolumn{2}{c}{ Without Coagulation } \\
\hline Mixing State & External & Internal & External & Internal \\
\hline Total number $\left(\# \mathrm{~cm}^{-3}\right)$ & $5.08 \times 10^{4}$ & $5.86 \times 10^{4}$ & $1.46 \times 10^{5}$ & $1.46 \times 10^{5}$ \\
Total mass $\left(\mu \mathrm{g} \mathrm{m}^{-3}\right)$ & 58.2 & 60.0 & 56.2 & 60.0 \\
INERT $\left(\mu \mathrm{g} \mathrm{m}^{-3}\right)$ & 30.0 & 30.0 & 30.0 & 30.0 \\
PO1 $\left(\mu \mathrm{g} \mathrm{m}^{-3}\right)$ & 7.50 & 7.50 & 7.50 & 7.50 \\
PO2 $\left(\mu \mathrm{g} \mathrm{m}^{-3}\right)$ & 7.50 & 7.50 & 7.50 & 7.50 \\
SV1 $\left(\mu \mathrm{g} \mathrm{m}^{-3}\right)$ & 4.61 & 4.62 & 4.09 & 4.62 \\
SV2 $\left(\mu \mathrm{g} \mathrm{m}^{-3}\right)$ & 8.62 & 10.4 & 7.14 & 10.4 \\
\hline
\end{tabular}

are identical to the initial population used in the internal mixture case, with the same total mass and component distribution. During simulations, partitioning of SV1 and SV2 and coagulation alter the composition of these initial populations. When the composition changes beyond the specified limits for a given population, the aerosol is moved to one of the other compositional populations. In the base case scenario, the mixing threshold criterion, $c$, is set at 0.1 for all components. The influence of the mixing threshold value on simulation results is discussed further in Sect. 4.3.

\section{Simulation results}

\subsection{Mixing state}

Simulations were performed with the base case scenario for both an external and an internal mixture. The predicted distributions for external and internal mixtures are shown in Fig. 3 and Table 3. In both cases, the aerosol distribution shows growth to larger particle sizes and the addition of semivolatile compounds, but the specific growth behavior for external and internal mixture simulations is different. Since both simulations used the same base case scenario to define emissions and other input parameters, differences in model results are entirely attributable to the assumed mixing state.

When semivolatile compounds, SV1 and SV2, partition to the aerosol phase, their partitioning behavior can be strongly influenced by aerosol phase interactions. Because of their similar polarity, nonpolar SV1 prefers to condense on particles composed of nonpolar PO1, while polar SV2 is more likely to partition to polar PO2. When the aerosol is treated as an internal mixture, this partitioning difference between SV1 and SV2 is not seen, because all particles have the same percentage of PO1 and PO2. As a result, for an internal mixture particles of different sizes have the exact same composition, as shown in Fig. 3b. Because of its higher partitioning coefficient, more SV2 condenses to the aerosol phase than SV1, and there is a higher percentage of SV2 in each size section.

For an external mixture, composition is not uniform, but varies with particle size. The total aerosol distribution, summed from the individual aerosol populations, with accompanying pie plots of the composition in each size section, is shown in Fig. 3a. Because more SV2 condenses to the particle phase than SV1, and SV2 partitions preferentially to $\mathrm{PO} 2$, particles made of $\mathrm{PO} 2$ grow slightly faster than those of PO1. As a result, higher fractions of PO2 and SV2 occur in the largest size sections. In smaller size sections, INERT dominates because no semivolatile organic compounds condense on it, and the movement of INERT to larger sizes only occurs through coagulation between particles.

The external mixture also causes a net reduction in semivolatile organic aerosol for these simulations. As shown in Table 3, the mass of component SV2 in the aerosol phase 

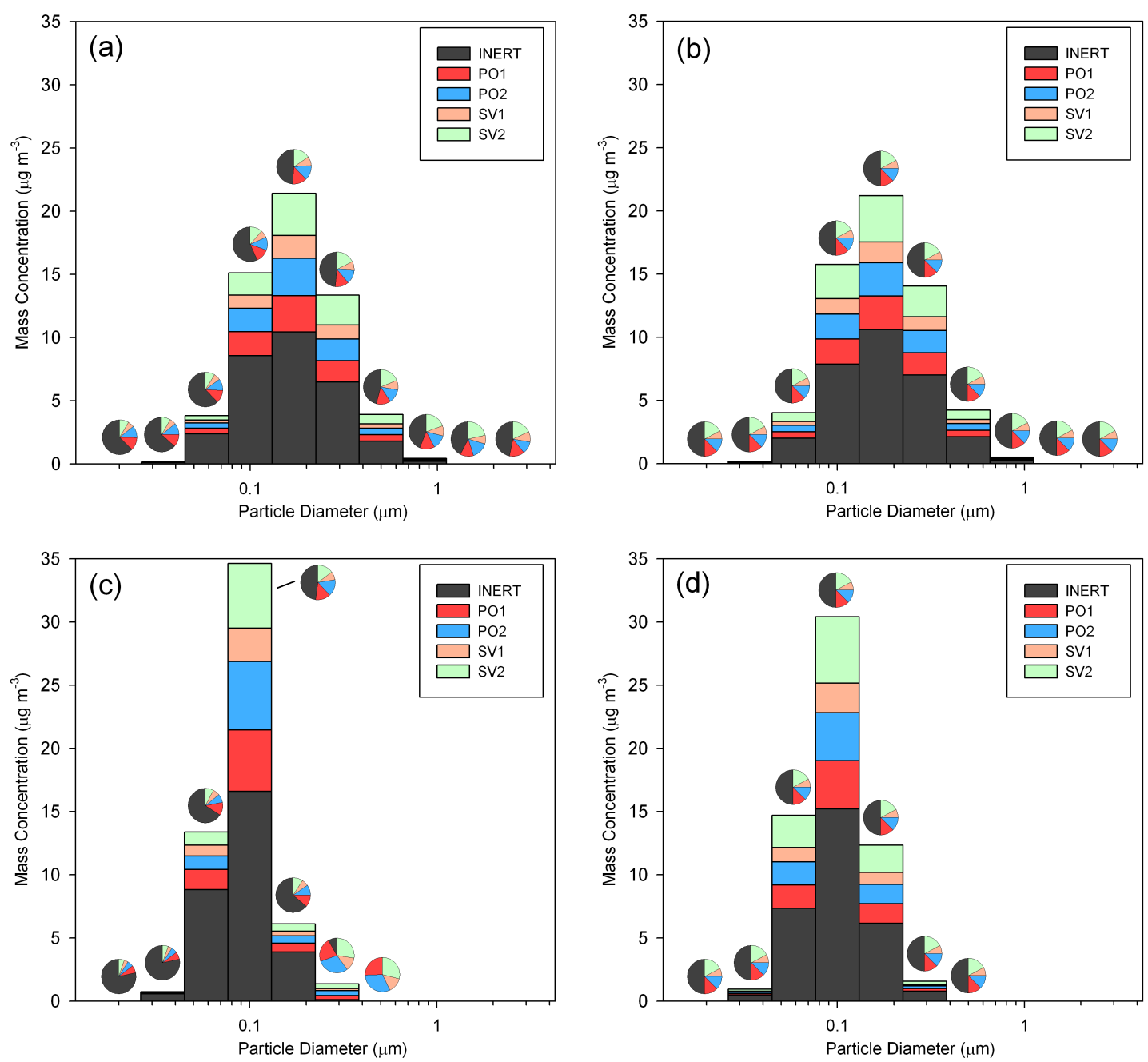

Fig. 3. Size and component distributions at $t=188 \mathrm{~h}$ for base case scenario simulations. (a) external mixture with coagulation, (b) internal mixture with coagulation, (c) external mixture without coagulation, (d) internal mixture without coagulation.

is $10.4 \mu \mathrm{g} \mathrm{m}^{-3}$ for the internal mixture, but only $8.62 \mu \mathrm{g} \mathrm{m}^{-3}$ for the external mixture. For SV1, the aerosol phase concentration remains essentially unchanged at $4.6 \mu \mathrm{g} \mathrm{m}^{-3}$. The partitioning behavior of these compounds depends on the amount of absorbing mass available and the chemical composition of the absorbing phase(s). For an internal mixture, there is a single uniform absorbing phase containing both nonpolar PO1 and polar PO2. This mixture of nonpolar and polar organics is not an optimum absorption medium for either nonpolar SV1 or polar SV2, but there is a relatively large absorbing mass available. For the external mixture simulation, $\mathrm{PO} 1$ and $\mathrm{PO} 2$ are largely segregated into different particle populations. Relative to the internal PO1/PO2 mixture, the PO1-dominated populations in the external mixture increase partitioning of SV1 and reduce partitioning of SV2, while the PO2-dominated populations have the reverse effect, decreasing SV1 partitioning and increasing SV2 partitioning. The net result of these competing effects is the ob- served decrease in partitioning to the aerosol phase for SV2 and the unchanged partitioning for SV1. As seen in this case, the effect of mixing state on partitioning is complex and will depend on the specific semivolatile compounds and absorbing aerosol phases present.

Despite these changes in partitioning, the overall aerosol size distribution is similar for both external and internal mixtures, as shown in Fig. 3a-b. A major fraction of both distributions is composed of the nonvolatile model species INERT, $\mathrm{PO} 1$, and PO2. The differences in growth due to SV1 and SV2 partitioning that are seen in total and compound mass concentrations are not large enough to significantly alter the size distribution. Scenarios where semivolatile inorganic compounds and water vapor also partition to the aerosol phase might result in a larger impact of mixing state on the overall size distribution, but semivolatile organics alone are not expected to contribute enough mass to produce such an effect. 
Table 4. Major populations* formed at $t=188 \mathrm{~h}$ in base case and simplified scenarios with an external mixture.

\begin{tabular}{|c|c|c|c|c|c|c|c|c|c|}
\hline \multirow{3}{*}{ Index } & \multirow{2}{*}{\multicolumn{5}{|c|}{ Components }} & \multicolumn{2}{|c|}{ Base Case } & \multicolumn{2}{|c|}{ Simplified } \\
\hline & & & & & & Mass & Number & Mass & Number \\
\hline & INERT & PO1 & $\mathrm{PO} 2$ & SV1 & SV1 & $\left(\mu \mathrm{g} \mathrm{m}^{-3}\right)$ & $\left(\# \mathrm{~cm}^{-3}\right)$ & $\left(\mu \mathrm{g} \mathrm{m}^{-3}\right)$ & $\left(\# \mathrm{~cm}^{-3}\right)$ \\
\hline 1 & + & & & & & 16.9 & $2.7 \times 10^{4}$ & 16.2 & $2.7 \times 10^{4}$ \\
\hline 2 & & + & & + & & 3.6 & $5.8 \times 10^{3}$ & 3.6 & $5.7 \times 10^{3}$ \\
\hline 3 & & & + & & + & 4.1 & $5.8 \times 10^{3}$ & 4.2 & $5.9 \times 10^{3}$ \\
\hline 4 & + & + & & + & & 9.4 & $4.4 \times 10^{3}$ & 10.4 & $4.5 \times 10^{3}$ \\
\hline $4 b$ & + & + & & + & + & 1.1 & $2.8 \times 10^{2}$ & & \\
\hline 5 & + & & + & & + & 12.9 & $5.0 \times 10^{3}$ & 13.8 & $5.1 \times 10^{3}$ \\
\hline 6 & & + & + & + & + & 0.9 & $4.3 \times 10^{2}$ & 1.0 & $5.1 \times 10^{2}$ \\
\hline 7 & + & + & + & + & + & 3.0 & $4.9 \times 10^{2}$ & 9.0 & $2.1 \times 10^{3}$ \\
\hline $7 a$ & + & + & + & & + & 6.0 & $1.2 \times 10^{3}$ & & \\
\hline
\end{tabular}

* At least $0.3 \mu \mathrm{g} \mathrm{m}^{-3}$ total mass in population.

The other clear difference between internal and external mixture simulations, is that the predicted composition of individual particles changes drastically. Unlike internally mixed particles where there is only 1 population with uniform composition, the external mixture simulations predict the formation of 9 major compositional populations as shown in Table 4 and Fig. 4a. Other mixture populations are also created by the model, but their mass and number concentrations are small enough to be negligible and are not reported here. Semivolatile partitioning and coagulation affect the original 3 populations in different ways. The original PO1 population disappears, and most PO1 mass is found in population 2 (PO1/SV1 mixture), and population 4 (PO1/SV1/INERT mixture). The PO1/SV1 mixture is formed when SV1 partitions to emitted PO1, while the PO1/SV1/INERT mixture is produced from both coagulation between PO1/SV1 and INERT populations or partitioning of SV1 to a coagulation-formed PO1/INERT population. Similarly, there is no $\mathrm{PO} 2$ population left, as most $\mathrm{PO} 2$ shifts to distributions 3 (PO2/SV2 mixture) and 5 (PO2/SV2/INERT mixture). The original INERT population, however, still exists with significant mass and number concentrations. Unlike the organic populations, SV1 and SV2 do not partition to INERT in this model, and mixing between INERT and the organic components occurs almost entirely through coagulation. Because there is a constant emission source of INERT particles and coagulation occurs on a relatively long time scale, only a fraction of the INERT component is transferred to other populations.

The other populations that are produced, most notably populations 7 (PO1/PO2/SV1/SV2/INERT) and 7a (PO1/PO2/SV2/INERT) which contain significant amounts of all (or most all) the aerosol components, arise from coagulation between already mixed populations. Since these populations are not formed directly from the original pure (a)

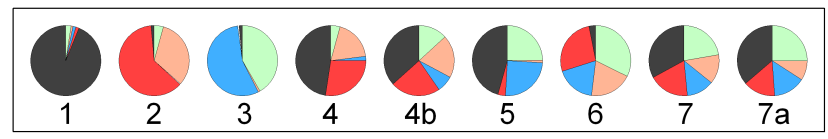

(b)

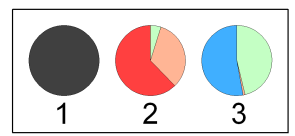

Fig. 4. Compositional plots for external mixture aerosol populations formed at $t=188 \mathrm{~h}$ in base case scenario simulations. (a) with coagulation, (b) without coagulation.

populations, they have somewhat lower mass and number concentrations. It is also important to recognize that once the simulation begins all populations contain at least small amounts of every component. For example, population 5 is labeled as a mixture of PO2, SV2, and INERT, but as can be seen in Fig. 4a, it also includes PO1 and SV1 at levels below the $10 \%$ mixing threshold. In some cases, subpopulations, such as $4 \mathrm{~b}$ (PO1/SV1/SV2/INERT) and $7 \mathrm{a}(\mathrm{PO} 1 / \mathrm{PO} 2 / \mathrm{SV} 2 / \mathrm{INERT})$, are denoted that contain either slightly more (SV2 in population $4 b$ ) or less (SV1 in population 7a) of one component.

The different populations show distinct size distributions that reflect the degree of mixing and growth from coagulation and condensation. As shown in Fig. 5a the less mixed populations, 1 (INERT), 2 (PO1/SV1), and 3 (PO2/SV2), contain smaller particles. The mean particle diameter of each of these populations is approximately $0.1 \mu \mathrm{m}$, which is only slightly larger than the emitted populations. Moderately mixed populations, including 
Table 5. Populations formed at $t=188 \mathrm{~h}$ in base case external mixture simulation without coagulation.

\begin{tabular}{|c|c|c|c|c|c|c|c|}
\hline \multirow[t]{2}{*}{ Index } & \multicolumn{5}{|c|}{ Components } & \multirow[t]{2}{*}{ Mass $\left(\mu \mathrm{g} \mathrm{m}^{-3}\right)$} & \multirow[t]{2}{*}{ Number $\left(\# \mathrm{~cm}^{-3}\right)$} \\
\hline & INERT & PO1 & $\mathrm{PO} 2$ & SV1 & SV2 & & \\
\hline 1 & + & & & & & 30.0 & $9.8 \times 10^{4}$ \\
\hline 2 & & + & & + & & 12.1 & $2.4 \times 10^{4}$ \\
\hline 3 & & & + & & + & 14.2 & $2.4 \times 10^{4}$ \\
\hline
\end{tabular}

Table 6. Major populations* formed at $t=188 \mathrm{~h}$ in base case external mixture simulations with different mixing criteria.

\begin{tabular}{|c|c|c|c|c|c|c|c|c|c|}
\hline \multirow[t]{2}{*}{ Index } & \multicolumn{5}{|c|}{ Components } & \multicolumn{4}{|c|}{ Mass concentration $\left(\mu \mathrm{g} \mathrm{m}^{-3}\right)$} \\
\hline & INERT & PO1 & $\mathrm{PO} 2$ & SV1 & SV2 & $c=0.02$ & $c=0.05$ & $c=0.1$ & $c=0.2$ \\
\hline 1 & + & & & & & 11.27 & 13.13 & 16.87 & 29.11 \\
\hline $1 \mathrm{a}$ & + & & & & + & 0.05 & 0.10 & 0.05 & 0.33 \\
\hline 2 & & + & & + & & 0.16 & 3.03 & 3.57 & 6.37 \\
\hline $2 \mathrm{a}$ & & + & & + & + & 2.61 & 0.03 & 0.08 & 0.00 \\
\hline $2 b$ & & + & & & + & 0.00 & 0.00 & 0.00 & 0.37 \\
\hline 3 & & & + & & + & 3.04 & 3.51 & 4.10 & 7.09 \\
\hline 4 & + & + & & + & & 5.27 & 8.28 & 9.41 & 6.57 \\
\hline $4 a$ & + & + & & & & 0.00 & 0.00 & 0.02 & 0.52 \\
\hline $4 b$ & + & + & & + & + & 2.01 & 0.87 & 1.07 & 0.00 \\
\hline 5 & + & & + & & + & 7.54 & 9.35 & 12.94 & 7.29 \\
\hline 6 & & + & + & + & + & 0.88 & 0.93 & 0.85 & - \\
\hline $6 a$ & & + & + & & + & 0.00 & 0.14 & 0.26 & 0.79 \\
\hline 7 & + & + & + & + & + & 24.58 & 16.12 & 3.00 & - \\
\hline $7 \mathrm{a}$ & + & + & + & & + & 0.85 & 2.72 & 5.97 & 0.00 \\
\hline \multirow[t]{2}{*}{ All Others } & \multirow{2}{*}{\multicolumn{3}{|c|}{ Total mass }} & & & 0.00 & 0.02 & 0.04 & 0.03 \\
\hline & & & & & & 58.3 & 58.2 & 58.2 & 58.5 \\
\hline
\end{tabular}

* At least $0.3 \mu \mathrm{g} \mathrm{m}^{-3}$ total mass in population for at least one mixing criteria.

4 (PO1/SV1/INERT), 4b (PO1/SV1/SV2/INERT), 5 (PO2/SV2/INERT), and 6 (PO1/PO2/SV1/SV2) are slightly larger in size, with mean diameters approaching $0.2 \mu \mathrm{m}$. Highly mixed populations 7 (PO1/PO2/SV1/SV2/INERT) and $7 \mathrm{a}(\mathrm{PO} 1 / \mathrm{PO} 2 / \mathrm{SV} 2 / \mathrm{INERT})$ exhibit the most growth with a combined mean diameter of approximately $0.3 \mu \mathrm{m}$. It is this difference in particle size for compositional populations that produces the size varying average particle composition observed in Fig. 3a.

\subsection{Coagulation}

Internal and external mixture simulations of the base case scenario were also run with coagulation processes turned off in the model. Comparison of results for simulations without coagulation (Figs. 3c-d, 5b and Tables 3 and 5) with simulations including coagulation (Figs. 3a-b, 5a and Tables 3 and 4) highlight the impact of coagulation on particle growth and mixing. As expected, coagulation lowers the number of particles, with both external and internal mixture total number concentrations reduced to approximately one third of their values without coagulation. At the same time, particle growth due to coagulation leads to broader size distributions with more particles growing to the larger size sections, as seen in Figs. 3 and 5. Total particle mass, however, is not affected as dramatically. The internal mixture simulations with and without coagulation both result in a total mass of $60.0 \mu \mathrm{g} \mathrm{m}^{-3}$. In contrast, the external mixture results in $58.2 \mu \mathrm{g} \mathrm{m}^{-3}$ with coagulation and $56.2 \mu \mathrm{g} \mathrm{m}^{-3}$ without. Differences in total mass are due solely to changes in the partitioning of SV1 and SV2 as emission and deposition of the primary species are the same in all simulations. As discussed in the previous section, for this simulation partitioning is reduced as the aerosol becomes more externally mixed. The external mixture simulation without coagulation is the most externally mixed scenario and, consequently, predicts the lowest amount of semivolatile aerosol formation. 
The most significant difference between the simulation results with or without coagulation is the number of compositional populations. For the internal mixture, there is always only one population, but for the external mixture, coagulation causes the number of major populations to increase from 3 to 9. When coagulation is neglected, there are no interactions between different populations, the initial 3 populations grow independently, and no new populations are formed. Since SV1 partitions almost exclusively to PO1 and SV2 to PO2, the populations undergo only partial mixing. As shown in Fig. 4b, one population contains only INERT, another mainly $\mathrm{PO} 1$ and SV1, and the third is composed of $\mathrm{PO} 2$ and SV2. When the simulation includes coagulation, however, the initial components are able to mix with each other and many new populations are created. As seen in Fig. $4 \mathrm{a}$, a wide range of compositional mixtures is produced, with most populations containing at least minor amounts of each component. These more mixed populations that result from coagulation also grow to larger sizes as seen in Fig. 5. Without coagulation (Fig. 5b), the 3 compositional populations undergo only minor growth due to condensation. With coagulation (Fig. 5a), however, the compositional populations formed via coagulation grow to larger sizes, and population 7, which results from coagulation of all three initial populations, is the largest of all.

\subsection{Mixing criterion}

In the base case simulations, a mass fraction of 0.1 was arbitrarily defined as the threshold for determining whether a component should be considered part of a mixture. In order to investigate the influence of this mixing threshold on simulation results, the base case scenario was run with the mixing threshold criterion, $c$, set as $0.02,0.05,0.1$ and 0.2 , and the simulated aerosol size and composition distributions were compared.

Simulation results showed that the size and composition distributions are generally insensitive to the choice of mixing criteria. In all cases, the composition distributions are similar, with a high percentage of INERT in smaller size particles while the larger particles have a higher fraction of organic compounds.

Where the specified mixing criterion does have a great effect is on the mass concentrations of the individual compositional populations, as shown in Table 6. When $c$ is set to a low value of 0.02 , population 7 (a mixture of all five components) has the highest mass concentration, and most of the mass is found in populations that are mixtures of organics and INERT. As the mixing criterion increases, mass shifts to the less well-mixed populations because of the higher threshold for defining what constitutes a mixture. When the threshold is set as high as 0.2 , population 7 contains no particles, mass is concentrated in a smaller number of major populations, and the majority of particle mass is found in population 1 (the "pure" INERT mixture).
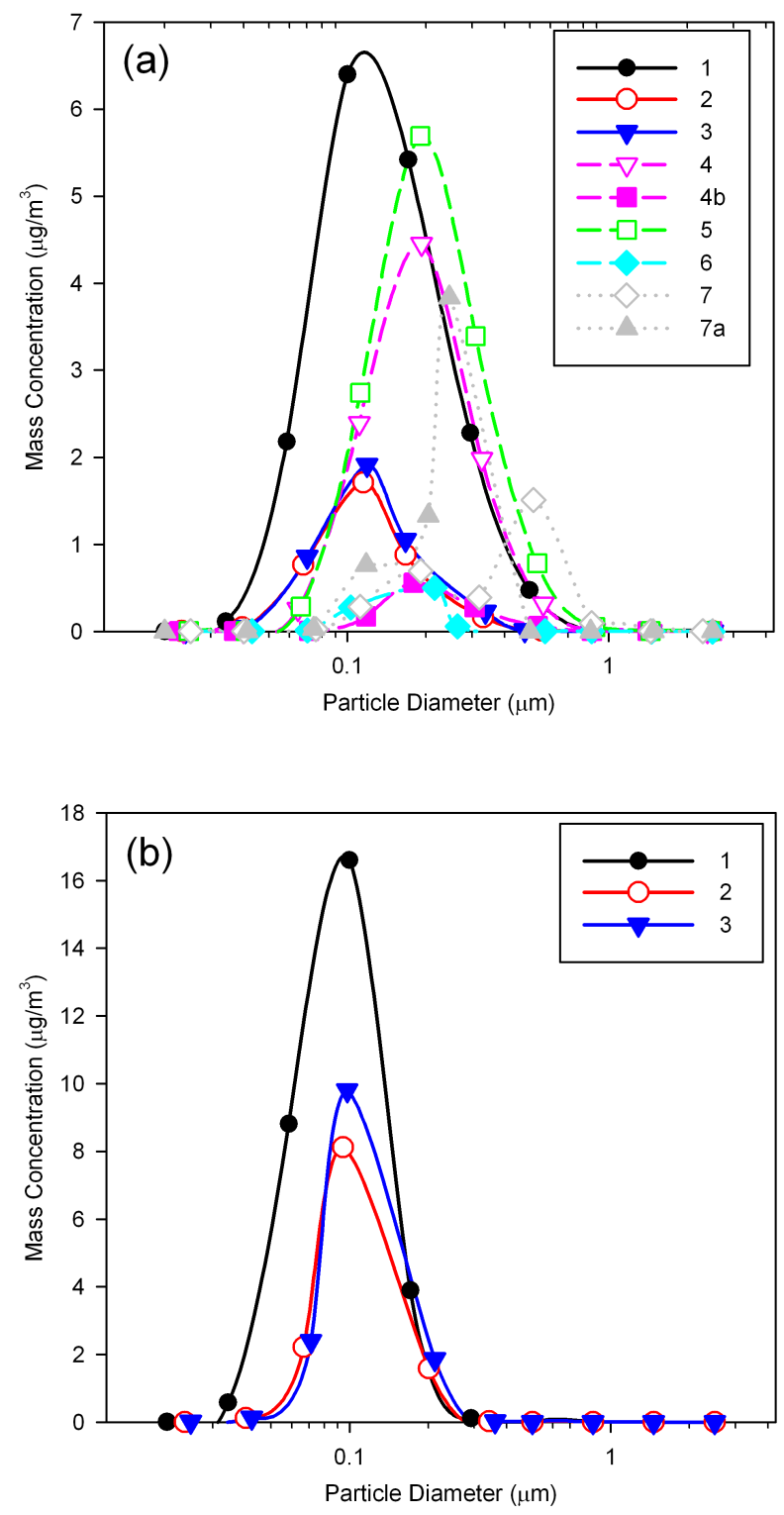

Fig. 5. Size distributions for external mixture aerosol populations formed at $t=188 \mathrm{~h}$ in base case scenario simulations. (a) with coagulation, (b) without coagulation.

These results from the high $(c=0.2)$ and low $(c=0.02)$ mixing criterion simulations show the limiting behavior that can be expected from the model. When the mixing criterion is small, only a small amount of a component needs to be added to form a mixture, leading to a large number of "mixed" populations even though they may in reality be dominated by a single component. In the extreme case, where $c$ approaches zero, all of the mass will move to the completely mixed population as soon as condensation or coagulation occurs. At the other limit, a large value for the mixing criterion will inhibit the formation of mixed populations, and only a few major populations will be produced. By forcing components into 
Table 7. Comparison of computational time requirements for different model configurations.

\begin{tabular}{lll}
\hline $\begin{array}{l}\text { Simulation } \\
\text { Scenario }\end{array}$ & CPU time & $\begin{array}{l}\text { Possible } \\
\text { Occupied Bins }\end{array}$ \\
\hline Base Case* $^{*}$ & 990 & 310 \\
No coagulation & 55 & 90 \\
Internal mixture & 2.1 & 10 \\
Internal mixture, no coagulation & 1.6 & 10 \\
Simplified external mixture & 35 & 70 \\
5 size sections & 200 & 155 \\
8 size sections & 580 & 248 \\
16 size sections & 3200 & 496 \\
$c=0.20$ & 950 & 310 \\
$c=0.05$ & 950 & 310 \\
$c=0.01$ & 900 & 310 \\
\hline
\end{tabular}

* Base Case=external mixture, 5 components, 5 compounds, 10 size sections, $c=0.10$.

the less mixed populations, the intended definition of an external mixture will again be circumvented. Thus, to produce meaningful results, a reasonable value for the mixing criterion must be defined.

The definition of mixing criterion, $c$, depends on the system being modeled, and will be affected by the number, type, and concentration of components considered. For the current simulation system, the predicted distribution for $c=0.1$ appears to capture reasonably well the range of mixtures present. For more complex systems, where there is a much larger number of components, the mixing criterion would need to be much smaller, to reveal the effect of all the components. Additionally, when individual components are present at different concentrations it may be advantageous to specify a different mixing threshold for each component instead of using a common value for all components. Understanding how to best define mixing criteria values for different systems is a focus of our ongoing research.

\subsection{Controlling computational costs}

Accounting for multiple compositional populations and their interactions results in higher computational costs than for a simple internal mixture which contains only a single population of particles. As shown in Table 7, the base case external mixture simulation required approximately 500 times more computational time than the corresponding internal mixture simulation. Computational demand in the base case was due almost entirely to coagulation and gas-particle partitioning calculations, which accounted for approximately $75 \%$ and $25 \%$, respectively, of total cpu time. Computational time was roughly proportional to the square of the number of occupied particle bins in the model. With 31 compositional populations and 10 size sections, the base case has 310 bins, in contrast to an internal mixture that has only 10 bins for a single population. For external mixture simulations without coagulation, there are only 9 possible populations and cpu time was greatly reduced. Varying the number of size sections used to represent the particle size distribution also showed corresponding changes in cpu times. To a lesser degree, convergence criteria for the coagulation and condensation routines also had noticeable effects on computational costs, with tighter tolerances generally, but not uniformly, increasing computation time. However, changing the mixing threshold criteria, $c$, had little effect on computational demands. Mass shifted between particle populations as $c$ changed, but the total number of occupied bins for which calculations were performed remained relatively unchanged.

The computational times listed in Table 7 provide a qualitative picture of the level of detail that is computationally feasible. The current model formulation has undergone only limited optimization to minimize cpu times. The condensation routine uses the LSODES sparse matrix ODE solver (Hindmarsh, 1983), which provides a factor of 500 improvement over the basic LSODE solver. Other solvers that use multiple time step methods have recently been developed for atmospheric aerosol systems (Zhang and Wexler, 2006; Zaveri et al., 2008) and may be able to provide even greater efficiency. The coagulation routine skips empty bins when calculating coagulation rates, but there remains potential for further optimization. Even with additional improvements, however, the total number of components used will remain the primary factor controlling computational costs. For example, a 10 component mixture, with 10 size sections, would form 1023 possible populations, 10230 bins, and require 1000 times more cpu time than the base case with 5 components. Computational limitations aside, such a large number of bins would make the analysis of model results extremely difficult. Therefore, for both computational and practical terms it is important to limit the number of bins used.

Computational demands can be reduced by grouping individual model compounds together into a smaller number of components that define the mixture. For example, in the base case scenario, the five model compounds (INERT, PO1, PO2, SV1, SV2) can be represented by three mixture components (INERT, POLAR, NONPOLAR) with PO1 and SV1 grouped together as NONPOLAR, PO2 and SV2 grouped as POLAR, and INERT forming its own component. Compositional populations are defined based on the three components, but within the model the five compounds and their individual concentrations are still tracked independently for calculating emissions, partitioning, and other model processes. The simplified external mixture requires only 7 compositional populations, reducing computational time by a factor of 30 . Such a mixture definition not only improves computational efficiency but also makes physical sense. As seen in Table 4, the polar organics in this scenario are always present together, as are the nonpolar organics, with the 7 populations defined by the 3-component simplified mixture corresponding directly 
to the 7 primary populations formed by the 5-component base case mixture.

Simulations were conducted using this simplified 3component mixture with the mixing threshold criteria, $c$, set at 0.1 for INERT and 0.2 for POLAR and NONPOLAR. Total and compound mass concentrations and total number concentrations with the simplified external mixture definition are the same as for the base case. The overall size distribution is also nearly identical to the base case results, with only a slight shift of mass between the $0.5 \mu \mathrm{m}$ and $0.3 \mu \mathrm{m}$ size sections. Table 4 compares the simplified mixture and base case mixture predictions of mass and number concentrations for each of the compositional populations. The simplified mixture combines base case populations 4 and $4 \mathrm{~b}$ together and 7 and 7 a together, and mass is slightly higher in population 5 and lower in population 1 , but otherwise the simulations results are very similar for all populations.

When reducing the number of components in this way the choice of which compounds to group together can be determined beforehand when information on the types of mixtures that are likely to form is available, or can be specified after preliminary runs with a detailed mixture model. Other mixture definitions besides the DAMS representation could also be used as long as all possible compositions are assigned to some unique population so that any particle that is formed can be placed in a bin.

\section{Conclusions}

A new modeling method for describing externally mixed particles, the Detailed Aerosol Mixing State (DAMS) representation, has been developed. In this method, aerosol particles are classified by both size and composition, with a compositional population defined for every combination of aerosol mixture components and these populations divided into size sections. A mixing criterion is used to define boundaries between compositional populations, allowing DAMS to distinguish between particles that are truly mixed and ones that contain only small amounts of other components. The DAMS approach has been incorporated in a Lagrangian box model together with condensational growth and coagulation routines developed for use with the new representation.

Model results demonstrate that interactions between aerosol mixing state, semivolatile partitioning, and coagulation are significant. The assumption of aerosol mixing state affects the amount and types of semivolatile organics that partition to the available aerosol phases. And since the partitioning of semivolatile species is influenced by aerosolphase composition, the compositionally distinct aerosol populations in an external mixture exhibit different growth rates, producing a more size-varying composition compared to internal mixtures.

Both coagulation and condensation contribute to the mixing of aerosol particles. Condensation of semivolatile com- pounds changes particle composition as a mixture of primary and secondary aerosol is formed. While semivolatile compounds may partition preferentially to particles of similar composition, they will condense to some extent on all particles present. Over time this has the effect of adding secondary aerosol mass to initially distinct populations, making them more similar in composition. Coagulation mixes together aerosol particles from different compositional populations and tends to move all particles to a single well-mixed population. Simulation results show that coagulation has a significant influence on aerosol evolution and should be included when modeling aerosol mixing state. The aerosol distribution produced by the combined effects of condensation, coagulation, and emissions is predicted to be a collection of multiple compositionally distinct populations that exists somewhere between the extremes of a strictly external or internal mixture.

The selection of mixing threshold criteria shows limited effects on the final size and compositional distribution of particles, but has a significant impact on the size and type of individual populations that compose the external mixture. Properly defining mixing criteria values depends on the number, type, and concentration of primary and secondary aerosol components present and on the aerosol properties targeted for study. Computational demands for external mixture modeling are significant and should be controlled by limiting the number of aerosol populations that exist in the model. This can be achieved by grouping together individual model compounds to define a smaller number of mixture components. Guidelines for defining external mixtures in more complex aerosol systems need to be investigated further.

Acknowledgements. We thank Mark Jacobson for providing the noniterative semi-implicit coagulation code on which the iterative fully implicit code in this study was based. We also thank the anonymous reviewers for their valuable comments, which helped to improve the quality of this article.

Edited by: G. McFiggans

\section{References}

Bhave, P. V., Kleeman, M. J., Allen, J. O., and Hughes, L. S.: Evaluation of an air quality model for the size and composition of source-oriented particle classes, Environ. Sci. Technol., 36, 2154-2163, 2002.

Binkowski, F. S. and Roselle, S. J.: Models-3 community multiscale air quality (CMAQ) model aerosol component 1. Model description, J. Geophys. Res., 108(D6), 4183, doi:10.1029/2001JD001409, 2003.

Bowman, F. M., Odum, J. R., Seinfeld, J. H., and Pandis, S. N.: Mathematical model for gas-particle partitioning of secondary organic aerosols, Atmos. Environ., 31, 3921-3931, 1997.

Bowman, F. M. and Karamalegos, A. M.: Estimated effects of composition on secondary organic aerosol mass concentrations, Environ. Sci. Technol., 36, 2701-2707, 2002. 
Bowman, F. M. and Melton, J. A.: Effect of activity coefficient models on predictions of secondary organic aerosol partitioning, J. Aerosol Sci., 35, 1415-1438, 2004.

Broekhuizen, K., Kumar, P. P., and Abbatt, J. P. D.: Partially soluble organics as cloud condensation nuclei: Role of trace soluble and surface active species, Geophys. Res. Lett., 31, L01107, doi:10.1029/2003GL018203, 2004.

Chow, J. C., Watson, J. G., Kuhns, H., Etyemezian, V., Lowenthal, D. H., Crow, D., Kohl, S. D., Engelbrecht, J. P., and Green, M. C.: Source profiles for industrial, mobile, and area sources in the Big Bend Regional Aerosol Visibility and Observational study, Chemosphere, 54, 185-208, 2004.

Chow, J. C., Watson, J. G., Lowenthal, D. H., Chen, L. W. A., Zielinska, B., Mazzoleni, L. R., and Magliano, K. L.: Evaluation of organic markers for chemical mass balance source apportionment at the Fresno Supersite, Atmos. Chem. Phys., 7, 17411754, 2007, http://www.atmos-chem-phys.net/7/1741/2007/.

Corrigan, C. E. and Novakov, T.: Cloud condensation nucleus activity of organic compounds: a laboratory study, Atmos. Environ., 33, 2661-2668, 1999.

FassiFihri, A., Suhre, K., and Rosset, R.: Internal and external mixing in atmospheric aerosols by coagulation: Impact on the optical and hygroscopic properties of the sulphate-soot system, Atmos. Environ., 31, 1393-1402, 1997.

Griffin, R. J., Nguyen, K., Dabdub, D., and Seinfeld, J. H.: A coupled hydrophobic-hydrophilic model for predicting secondary organic aerosol formation, J. Atmos. Chem., 44, 171-190, 2003.

Hasegawa, S. and Ohta, S.: Some measurements of the mixing state of soot-containing particles at urban and non-urban sites, Atmos. Environ., 36, 3899-3908, 2002.

Hindmarsh, A. C.: ODEPACK, A systemized collection of ODE solvers, in: Scientific Computing, edited by: Stepleman, R. S., Carver, M., Peskin, R., Ames, W. F., Vichnevetsky, R., NorthHolland, Amsterdam, 55-64, 1983.

Jacobson, M. Z., Turco, R. P., Jensen, E. J., and Toon, O. B.: Modeling coagulation among particles of different composition and size, Atmos. Environ., 28, 1327-1338, 1994.

Jacobson, M. Z.: Development and application of a new air pollution modeling system - II. Aerosol module structure and design, Atmos. Environ., 31, 131-144, 1997a.

Jacobson, M. Z.: Fundamentals of Atmospheric Modeling, Cambridge University, New York, 1997b.

Jacobson, M. Z.: Strong radiative heating due to the mixing state of black carbon in atmospheric aerosols, Nature, 409, 695-697, 2001.

Jacobson, M. Z.: Analysis of aerosol interactions with numerical techniques for solving coagulation, nucleation, condensation, dissolution, and reversible chemistry among multiple size distributions, J. Geophys. Res., 107(D19), 4366, doi:4310.1029/2001JD002044, 2002.

Jung, C. H. and Kim, Y. P.: Numerical estimation of the effects of condensation and coagulation on visibility using the moment method, J. Aerosol Sci., 37, 143-161, 2006.

Kleeman, M. J., Cass, G. R., and Eldering, A.: Modeling the airborne particle complex as a source-oriented external mixture, J. Geophys. Res., 102, 21355-21372, 1997.

Lesins, G., Chylek, P., and Lohmann, U.: A study of internal and external mixing scenarios and its effect on aerosol optical prop- erties and direct radiative forcing, J. Geophys. Res., 107(D10), 4094, doi:10.1029/2001JD000973, 2002.

Naoe, H. and Okada, K.: Mixing properties of submicrometer aerosol particles in the urban atmosphere - with regard to soot particles, Atmos. Environ., 35, 5765-5772, 2001.

Pankow, J. F.: An absorption model of the gas/aerosol partitioning involved in the formation of secondary organic aerosol, Atmos. Environ., 28, 189-193, 1994a.

Pankow, J. F.: An absorption model of gas/particle partitioning of organic compounds in the atmosphere, Atmos. Environ., 28, 185-188, 1994b.

Pun, B. K., Wu, S. Y., and Seigneur, C.: Contribution of biogenic emissions to the formation of ozone and particulate matter in the eastern united states, Environ. Sci. Technol., 36, 3586-3596, 2002.

Riemer, N., West, M., Zaveri, R. A., and Easter, R. C.: Simulating the evolution of soot mixing state with a particleresolved aerosol model, J. Geophys. Res., 114, D09202, doi:10.1029/2008JD011073, 2009.

Rogge, W. F., Hildemann, L. M., Mazurek, M. A., Cass, G. R., and Simoneit, B. R. T.: Sources of fine organic aerosol .2. Noncatalyst and catalyst-equipped automobiles and heavy-duty diesel trucks, Environ. Sci. Technol., 27, 636-651, 1993.

Schauer, J. J., Kleeman, M., Cass, G. R., and Simoneit, B. R. T.: Measurement of emissions from air pollution sources. 2 . C1 through C30 organic compounds from medium duty diesel trucks, Environ. Sci. Technol., 33, 1578-1587, 1999.

Schauer, J. J., Kleeman, M., Cass, G. R., and Simoneit, B. R. T.: Measurement of emissions from air pollution sources. 3. C1-C29 organic compounds from fireplace combustion of wood, Environ. Sci. Technol., 35, 1716-1728, 2001.

Seinfeld, J. H. and Pandis, S. N.: Atmospheric Chemistry and Physics: From Air Pollution to Global Change, 2nd ed., John Wiley, New York, 1998.

Sheehan, P. E. and Bowman, F. M.: Estimated effects of temperature on secondary organic aerosol concentrations, Environ. Sci. Technol., 35, 2129-2135, 2001.

Wexler, A. and Seinfeld, J. H.: The distribution of ammonium salts among a size and composition dispersed aerosol, Atmos. Environ., 24A, 1231-1246, 1990.

Yao, X., Lau, N. T., Fang, M., and Chan, C. K.: Realtime observation of the transformation of ultrafine atmospheric particle modes, Aerosol Sci. Technol. 39, 831-841, doi:10.1080/02786820500295248, 2005.

Ying, Q., Mysliwiec, M., and Kleeman, M. J.: Source apportionment of visibility impairment using a three-dimensional sourceoriented air quality model, Environ. Sci. Technol., 38, 10891101, 2004.

Ying, Q., Fraser, M. P., Griffin, R. J., Chen, J., and Kleeman, M. J.: Verification of a source-oriented externally mixed air quality model during a severe photochemical smog episode, Atmos. Environ., 41, 1521-1538, 2007.

Zaveri, R. A., Easter, R. C., Fast, J. D., and Peters, L. K.: Model for Simulating Aerosol Interactions and Chemistry (MOSAIC), J. Geophys. Res., 113, D13204, doi:10.1029/2007JD008782, 2008.

Zhang, K. M. and Wexler, A. S.: An asynchronous time-stepping (ATS) integrator for atmospheric applications: Aerosol dynamics, Atmos. Environ., 40, 4574-4588, 2006. 